\title{
Meta-analysis of Xiangsha Liujunzi Decoction in Treating Chronic Atrophic Gastritis
}

\author{
Yunhan Yin, Jian Song*, Wen Wen, Yuqing Xu, Yanan Ai \\ Shaanxi University of Traditional Chinese Medicine, Xianyang 712046, China \\ *Correspondence Author
}

\begin{abstract}
Objective: To evaluate the efficacy and safety of Xiangsha Liujunzi Decoction in the treatment of chronic atrophic gastritis (CAG). Methods: PubMed, Embase, Cochrane Library, CBM, VIP, Wanfang and CNKI databases were searched by computer from March 2021 to March 2021. Subject words combined with free words were used for retrieval, literature was screened according to inclusion and exclusion criteria, and quality evaluation and data extraction were carried out. After two researchers independently screened literature, extracted data and evaluated the bias risk of included studies, Meta-analysis was performed using RevMan 5.3 software. Results: A total of 31 RCTs were included, including 2898 patients. The results of Meta-analysis showed that the combined or separate use of Xiangsha Liujunzi Decoction could improve the total clinical effective rate and Hp negative conversion rate, reduce the recurrence rate and oxidative stress response, and improve the clinical symptoms such as stomachache, anorexia, loose stools, shortness of breath, laziness, and discomfort of fullness.
\end{abstract}

Keywords: Xiangsha Liujunzi Decoction, Chronic atrophic gastritis, Meta-analysis, Systematic evaluation, Randomized controlled trial. Abbreviations: CAG, Chronic atrophic gastritis; TCM, Traditional Chinese medicine; HP, Helicobacter pylori; SOD, Superoxide Dismutase; MDA, Malondialdehyde.

\section{Introduction}

Chronic atrophic gastritis (CAG) is a chronic gastritis that occurs locally or extensively in the gastric mucosa with atrophy and reduction of the number of inherent glands, thinning of the mucosa or thickening of the muscular layer of the membrane, accompanied by low gastric secretion in different degrees, and is prone to intestinal metaplasia[1,2]. Patients are prone to belching, upper abdominal fullness or dull pain, nausea and other clinical symptoms. Some patients have upper gastrointestinal bleeding and other symptoms when they are serious, which seriously affects the health level of patients[3,4]. A multicenter epidemiological survey in 2014 showed that the prevalence of CAG in China is currently high, about $25.8 \%$ [5]. CAG is associated with Helicobacter pylori infection, environmental factors and genetic factors, and Helicobacter pylori infection is the main cause of the disease[3,6]. With the progression of the disease, pathological changes such as mucosal muscular thickening and intestinal metaplasia may occur in CAG patients, which increases the risk of canceration. Therefore, active and effective treatment can not only improve the clinical symptoms of patients and improve the quality of life of patients, but also prevent the occurrence of cancer. The role of western medicine on CAG is single, and long-term use may produce adverse reactions, making treatment difficult.

Xiangsha Liujunzi Decoction (香砂六君子汤), alias Shensha Hewei Powder (参砂和胃散), originates from Luo Mei's famous ancient and modern medical prescription theory. It can be used to treat functional dyspepsia, gastroesophageal reflux disease, colon cancer and other diseases. This prescription has good curative effect, and is a classic prescription for the treatment of spleen and stomach diseases. Especially for the spleen and stomach qi deficiency incoordination, such as qi deficiency, qi inversion, qi stagnation and other syndromes are outstanding. Studies have shown that Xiangsha Liujunzi Decoction can significantly improve the clinical symptoms of CAG patients, and has less adverse drug reactions, so it is widely used in clinic. In recent years, many clinical studies have shown that Xiangsha Liujunzi Decoction combined with western medicine has remarkable therapeutic effect on CAG. However, there are differences in research programs and efficacy among clinical trials, resulting in uneven research results, which to some extent affects the promotion of Xiangsha Liujunzi Decoction. Therefore, this study collected randomized controlled trials of Xiangsha Liujunzi Decoction combined with western medicine in the treatment of CAG, objectively evaluated the efficacy and safety of Xiangsha Liujunzi Decoction in the treatment of $\mathrm{CAG}$, and provided reliable reference for the clinical application of Xiangsha Liujunzi Decoction in the treatment of CAG.

\section{Data and Methods}

\subsection{Inclusion Criteria}

\subsubsection{Research object}

Patients with definite diagnosis of CAG, age, source of cases are unlimited. Diagnostic criteria of western medicine : referring to the diagnostic and dialectical criteria formulated by the digestive system disease professional committee of the Chinese Association of Integrated Traditional Chinese and Western Medicine in 1990[7], "Guiding Principles of Clinical Research on New Drugs of Traditional Chinese Medicine"[8] or "Guidelines for Diagnosis and Treatment of Chronic Gastritis"[9] diagnostic criteria; diagnostic criteria of traditional Chinese medicine: refer to the diagnostic criteria of "Guiding Principles of Clinical Research on New Drugs of Traditional Chinese Medicine"[8] or "Consensus on TCM diagnosis and treatment of chronic atrophic gastritis"[10].

\subsubsection{Interventional measures Experimental group}

Xiangsha Liujunzi Decoction was used alone or on the basis of the control group. Control group: conventional drug 
treatment. Conventional drugs include western medicine and Chinese patent medicine. Western medicine mainly includes proton pump inhibitors, gastric mucosal protective agents, conventional anti-Hp antibiotics and gastric motility drugs. Chinese patent medicines mainly include Weifuchun Tablets, Moroccan and Weikangling Capsules.

\subsubsection{Outcome indicators}

(1) Clinical total effective rate, calculation method: total effective rate $=$ (markedly effective cases + effective cases $)$ / total cases $\times 100 \%$; (2) Recurrence rate; (3) Hp negative conversion ; (4) TCM symptom score: stomach pain symptom score, anorexia and anorexia symptom score, stool looseness symptom score, shortness of breath and laziness symptom score, and fullness discomfort symptom score; (5) Levels of oxidative stress indexes: Superoxide Dismutase (SOD) and Malondialdehyde (MDA)

\subsubsection{Type of study}

Xiangsha Liujunzi Decoction in the treatment of CAG randomized controlled trials (RCT), regardless of whether the blind method were included, language is not limited.
(1) Repeated published literature; (2) Incomplete or missing analysis data and unable to contact the original author; (3) Literature with inconsistent intervention measures; (4) Clinical case reports and summary papers on experience of famous veteran TCM doctors; (5) Animal experiments and other non-therapeutic literature; (6) Literature review; (7) Literature with improper statistical methods; (8) There was no literature that recognized diagnostic criteria or diagnostic methods.

\subsection{Literature Retrieval Strategy}

PubMed, Embase, Cochrane Library, CBM, VIP, Wanfang and CNKI databases were searched by computer. The starting time of retrieval literature is not limited, and the deadline is March 15, 2021. The retrieval is not restricted by language. Subject words combined with free words were used for retrieval, and the obtained literature was manually searched. Chinese search terms include chronic atrophic gastritis, Xiangsha Liujunzi decoction, Meta-analysis, system evaluation, etc. English search terms include chronic atrophic gastritis, Xiangsha Liujunzi decoction, Meta-analysis, systematic review, etc. Taking PubMed as an example, the search strategy is shown in Figure 1.

\subsubsection{Excluded criteria}

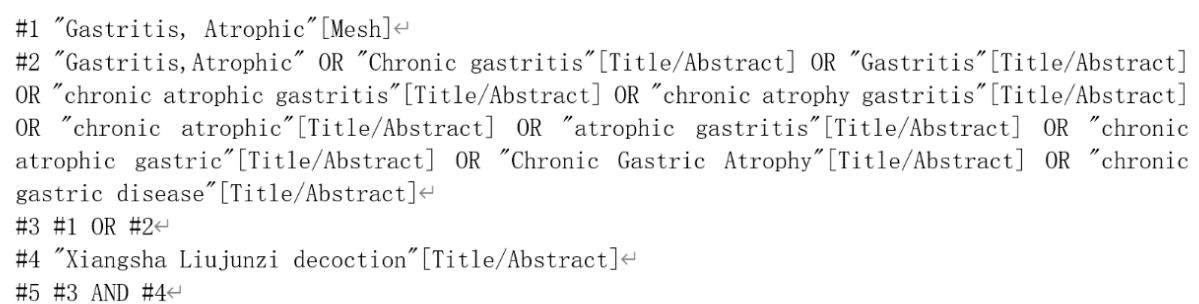

Figure 1: PubMed search strategy

\subsection{Literature Screening, Data Extraction}

Two qualified researchers (Yin Yunhan and Wen Wen) strictly followed the inclusion / exclusion criteria, independently screened literature, extracted data and cross-checked them. In case of disagreement, the third party (Ai Yanan) ruled. In the literature screening, the topics are first read. After excluding the obviously irrelevant literature, the summary and full text are further read to determine whether they are included. Contact the author of the original study by email or telephone, if necessary, to obtain undetermined but important information for this study. The content of data extraction includes: (1) Basic information included in the study : first author, publication time, research type, etc.; (2) Baseline characteristics and intervention measures of research objects; (3)Key elements of bias risk assessment; (4) Outcome indicators and measurement data.

\subsection{Bias Risk Assessment}

Two trained researchers (Yin Yunhan and $\mathrm{Xu}$ Yuqing) independently assessed the risk of bias of included studies, and cross-checked the results. The bias risk assessment was performed using RCT bias risk assessment tool recommended by Cochrane Manual 5.1.0.

\subsection{Statistical Analysis}

Meta-analysis was performed using RevMan5.3 software. The measurement data adopt standard mean difference (SMD) as the effect analysis statistics, and the binary variables adopt relative risk (RR) as the effect analysis statistics, with 95\% CI for each effect. The heterogeneity among the included research results was analyzed by $\chi 2$ test (the test level was $\alpha=0.1$ ), and the heterogeneity was quantitatively determined by $\mathrm{I}^{2}$. If there is no statistical heterogeneity among the research results, the fixed effect model is used for meta-analysis; if there is statistical heterogeneity among the research results, the sources of heterogeneity are further analyzed. After excluding the influence of obvious clinical heterogeneity, the random effect model is used for meta-analysis. The level of meta-analysis was set as $\alpha=0.05$. The significant clinical heterogeneity was treated by subgroup analysis or sensitivity analysis or only descriptive analysis.

\section{Results}

\subsection{Literature Screening}

A total of 1080 articles were retrieved, including 1074 articles in Chinese and 6 articles in English, including 3 articles in 
PubMed, 3 articles in Embase, 280 articles in CBM, 218 articles in VIP, 313 articles in WanFangDate and 263 articles in CNKI. After layer-by-layer screening, 31[11-41] Chinese literatures were finally included. The flow chart of literature screening is shown in Figure 2.

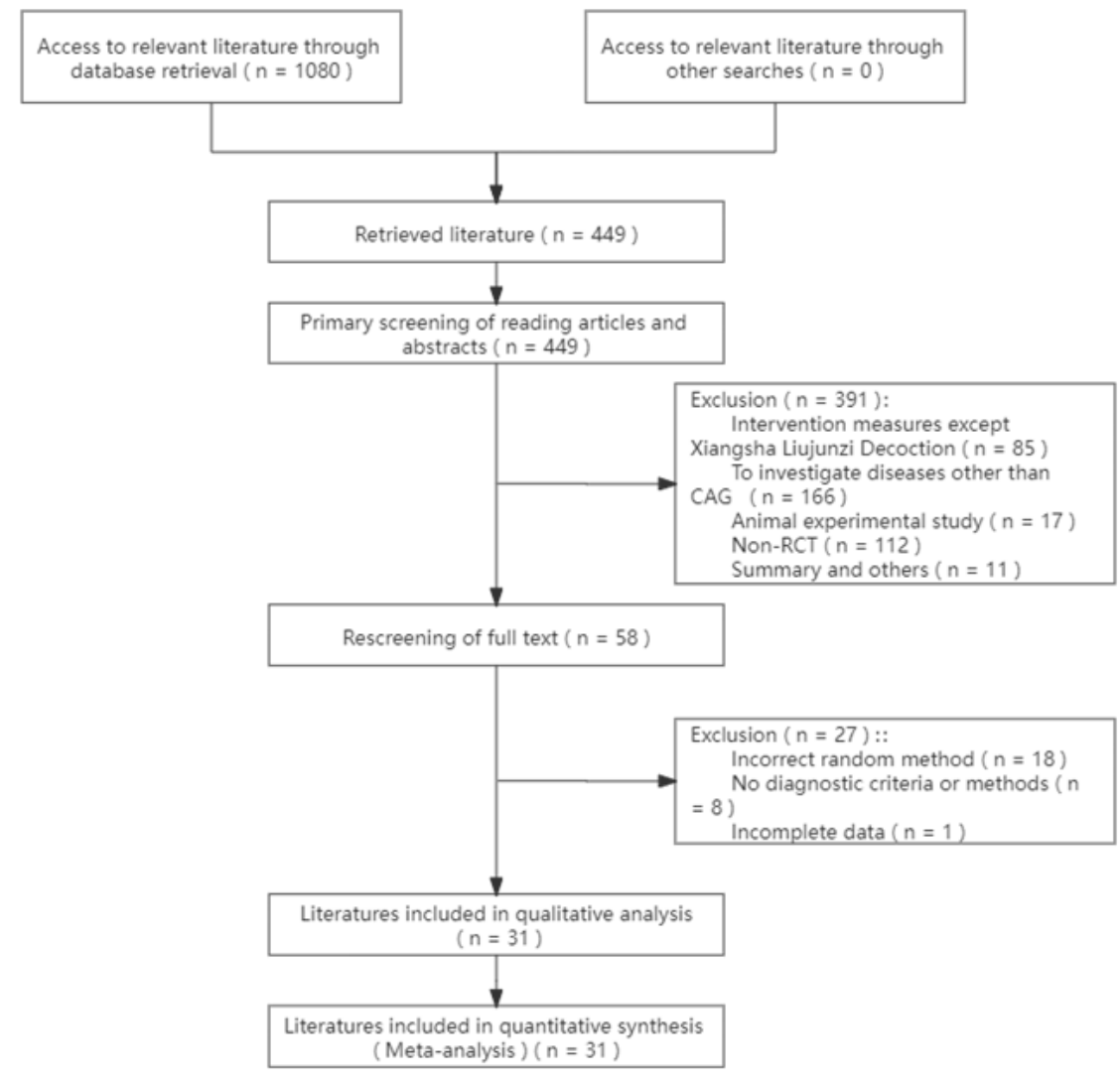

Figure 2: Literature screening process

Table 1: Basic information included in the study

\begin{tabular}{|c|c|c|c|c|c|c|c|c|c|}
\hline \multirow{2}{*}{ Eligible studies } & \multirow{2}{*}{ Research type } & \multicolumn{2}{|c|}{ Intervention measures } & \multicolumn{2}{|c|}{ Sample size } & \multicolumn{2}{|c|}{ Age / year } & \multirow{2}{*}{$\begin{array}{l}\text { Course } \\
\text { treatment }\end{array}$} & \multirow{2}{*}{$\begin{array}{l}\text { Outcome } \\
\text { index }\end{array}$} \\
\hline & & $\mathrm{T}$ & $\mathrm{C}$ & $\mathrm{T}$ & $\mathrm{C}$ & $\mathrm{T}$ & $\mathrm{C}$ & & \\
\hline $\begin{array}{l}\text { Chen Li } \\
2019^{[11]}\end{array}$ & $\begin{array}{l}\text { Double blind } \\
\text { screening } \\
\text { method }\end{array}$ & $\begin{array}{l}\text { Xiangsha Liujunzi } \\
\text { Decoction }\end{array}$ & $\begin{array}{l}\text { Chinese } \\
\text { patent } \\
\text { medicine }\end{array}$ & 40 & 40 & - & - & $6 \mathrm{~m}$ & (1)(4) \\
\hline $\begin{array}{l}\text { Du Jingfang } \\
2012^{[12]}\end{array}$ & Random & $\begin{array}{l}\text { Xiangsha Liujunzi } \\
\text { Decoction }\end{array}$ & $\begin{array}{l}\text { Western } \\
\text { medicine }\end{array}$ & 48 & 48 & $21-72$ & $22-67$ & $12 w$ & (1)(2) \\
\hline $\begin{array}{l}\text { Gao Meifu } \\
2017^{[13]}\end{array}$ & $\begin{array}{c}\text { Random } \\
\text { number table }\end{array}$ & $\begin{array}{l}\text { Xiangsha Liujunzi } \\
\text { Decoction }\end{array}$ & $\begin{array}{l}\text { Chinese } \\
\text { patent } \\
\text { medicine }\end{array}$ & 50 & 50 & $44.2 \pm 5.6$ & $43.4 \pm 4.9$ & $6 \mathrm{~m}$ & (1) \\
\hline $\begin{array}{l}\text { Lai Yu } \\
2012^{[14]}\end{array}$ & Random & $\begin{array}{l}\text { Xiangsha Liujunzi } \\
\text { Decoction }\end{array}$ & $\begin{array}{l}\text { Western } \\
\text { medicine }\end{array}$ & 30 & 30 & $50.60 \pm 6.31$ & $50.40 \pm 5.63$ & $3 \mathrm{~m}$ & (1)(4)(5)(6) (7) \\
\hline Lai Chan $2020^{[15]}$ & $\begin{array}{c}\text { Random } \\
\text { number table }\end{array}$ & $\begin{array}{l}\text { Xiangsha Liujunzi } \\
\text { Decoction }\end{array}$ & $\begin{array}{l}\text { Western } \\
\text { medicine }\end{array}$ & 65 & 65 & $49.86 \pm 6.14$ & $48.63 \pm 6.37$ & $8 w$ & $\begin{array}{c}\text { (1)(4) (5) (6) } 7 \text { (8) } \\
\text { (8) }\end{array}$ \\
\hline $\begin{array}{l}\text { Li Haifeng } \\
2014^{[16]}\end{array}$ & Random & $\begin{array}{l}\text { Xiangsha Liujunzi } \\
\text { Decoction }\end{array}$ & $\begin{array}{l}\text { Chinese } \\
\text { patent } \\
\text { medicine }\end{array}$ & 31 & 31 & $25-70$ & $23-71$ & $3 m$ & (1) (3) \\
\hline $\begin{array}{l}\text { Li Ning } \\
2020^{[17]}\end{array}$ & $\begin{array}{c}\text { Random } \\
\text { number table }\end{array}$ & $\begin{array}{c}\text { Xiangsha Liujunzi } \\
\text { Decoction + Control } \\
\text { group }\end{array}$ & $\begin{array}{l}\text { Western } \\
\text { medicine }\end{array}$ & 40 & 40 & $49.28 \pm 2.49$ & $49.54 \pm 2.45$ & $84 d$ & (1)(9)(10) \\
\hline $\begin{array}{l}\text { Liu Yiqiao } \\
2014^{[18]}\end{array}$ & Random & $\begin{array}{c}\text { Xiangsha Liujunzi } \\
\text { Decoction + Control } \\
\text { group }\end{array}$ & $\begin{array}{l}\text { Western } \\
\text { medicine }\end{array}$ & 30 & 30 & $51.5 \pm 9.9$ & $50.8 \pm 8.6$ & $8 w$ & (1)(2)(3) \\
\hline $\begin{array}{l}\text { Luo Yuanhan } \\
2007^{[19]}\end{array}$ & Random & $\begin{array}{l}\text { Xiangsha Liujunzi } \\
\text { Decoction }\end{array}$ & $\begin{array}{l}\text { Western } \\
\text { medicine }\end{array}$ & 38 & 30 & $28-69$ & $28-67$ & $3 \mathrm{~m}$ & (1) \\
\hline $\begin{array}{l}\text { Ma Shihua } \\
2011^{[20]}\end{array}$ & Random & $\begin{array}{l}\text { Xiangsha Liujunzi } \\
\text { Decoction }\end{array}$ & $\begin{array}{l}\text { Chinese } \\
\text { patent } \\
\text { medicine }\end{array}$ & 48 & 48 & $21-72$ & $22-67$ & $12 w$ & (1) \\
\hline $\begin{array}{l}\text { Mao Pingan } \\
2010^{[21]}\end{array}$ & Random & $\begin{array}{l}\text { Xiangsha Liujunzi } \\
\text { Decoction }\end{array}$ & $\begin{array}{l}\text { Chinese } \\
\text { patent } \\
\text { medicine }\end{array}$ & 45 & 31 & $43.15 \pm 9.21$ & $43.28 \pm 8.17$ & $6 m$ & (1) \\
\hline $\begin{array}{l}\text { Song Shixing } \\
2017^{[22]}\end{array}$ & $\begin{array}{l}\text { Drawing } \\
\text { method }\end{array}$ & $\begin{array}{l}\text { Xiangsha Liujunzi } \\
\text { Decoction }\end{array}$ & $\begin{array}{l}\text { Chinese } \\
\text { patent } \\
\text { medicine }\end{array}$ & 40 & 40 & $53.18 \pm 2.43$ & $53.29 \pm 2.51$ & $30 \mathrm{~d}$ & (1)(2)(3) \\
\hline $\begin{array}{c}\text { Su Xiaoyun } \\
2016^{[23]}\end{array}$ & Random & $\begin{array}{l}\text { Xiangsha Liujunzi } \\
\text { Decoction }\end{array}$ & $\begin{array}{l}\text { Western } \\
\text { medicine }\end{array}$ & 56 & 56 & $49.89 \pm 6.33$ & $49.93 \pm 6.32$ & $12 w$ & (1)(4)(5)(6)(7) \\
\hline
\end{tabular}




\begin{tabular}{|c|c|c|c|c|c|c|c|c|c|}
\hline $\begin{array}{l}\text { Tang Youming } \\
2010^{[24]}\end{array}$ & Random & $\begin{array}{l}\text { Xiangsha Liujunzi } \\
\text { Decoction }\end{array}$ & $\begin{array}{l}\text { Western } \\
\text { medicine }\end{array}$ & 75 & 75 & $36-70$ & $35-68$ & $60 d$ & $\begin{array}{l}\text { (8) } \\
\text { (1) (4) }(6) \\
\text { (8) }\end{array}$ \\
\hline $\begin{array}{l}\text { Wang Jianping } \\
2013^{[25]}\end{array}$ & Random & $\begin{array}{l}\text { Xiangsha Liujunzi } \\
\text { Decoction }\end{array}$ & $\begin{array}{l}\text { Chinese } \\
\text { patent } \\
\text { medicine }\end{array}$ & 70 & 70 & $50.6 \pm 7.3$ & $52.4 \pm 9.1$ & $6 \mathrm{~m}$ & (1) \\
\hline $\begin{array}{l}\text { Wang Musheng } \\
2008^{[26]}\end{array}$ & Random & $\begin{array}{c}\text { Xiangsha Liujunzi } \\
\text { Decoction + Control } \\
\text { group }\end{array}$ & $\begin{array}{l}\text { Western } \\
\text { medicine }\end{array}$ & 45 & 35 & $25-78$ & $24-76$ & $3 \mathrm{~m}$ & (1) \\
\hline $\begin{array}{l}\text { Wang Shunqin } \\
2020^{[27]}\end{array}$ & $\begin{array}{c}\text { Random } \\
\text { number table }\end{array}$ & $\begin{array}{c}\text { Xiangsha Liujunzi } \\
\text { Decoction + Control } \\
\text { group }\end{array}$ & $\begin{array}{l}\text { Western } \\
\text { medicine } \\
+ \text { Chinese } \\
\text { patent } \\
\text { medicine }\end{array}$ & 37 & 37 & $50.2 \pm 2.3$ & $49.8 \pm 2.2$ & $2 \mathrm{~m}$ & (1)(4) \\
\hline $\begin{array}{l}\text { Wang Xuemei } \\
2014^{[28]}\end{array}$ & Random & $\begin{array}{l}\text { Xiangsha Liujunzi } \\
\text { Decoction }\end{array}$ & $\begin{array}{l}\text { Western } \\
\text { medicine }\end{array}$ & 38 & 38 & $45.7 \pm 5.8$ & $44.9 \pm 5.9$ & $6 \mathrm{~m}$ & (1) \\
\hline $\begin{array}{l}\text { Wang Yawei } \\
2014^{[29]}\end{array}$ & Random & $\begin{array}{l}\text { Xiangsha Liujunzi } \\
\text { Decoction }\end{array}$ & $\begin{array}{l}\text { Chinese } \\
\text { patent } \\
\text { medicine }\end{array}$ & 69 & 69 & $51.89 \pm 6.76$ & $52.03 \pm 6.69$ & $1 \mathrm{~m}$ & (1)(2)(3) \\
\hline $\begin{array}{l}\text { Wen Liqiong } \\
2011^{[30]}\end{array}$ & Random & $\begin{array}{l}\text { Xiangsha Liujunzi } \\
\text { Decoction }\end{array}$ & $\begin{array}{l}\text { Chinese } \\
\text { patent } \\
\text { medicine }\end{array}$ & 120 & 120 & $32-69$ & $31-67$ & $3 \mathrm{~m}$ & (1) \\
\hline $\begin{array}{l}\text { Zhang } \\
\text { Changming } \\
2018^{[31]}\end{array}$ & $\begin{array}{c}\text { Random } \\
\text { number table }\end{array}$ & $\begin{array}{l}\text { Xiangsha Liujunzi } \\
\text { Decoction }\end{array}$ & $\begin{array}{l}\text { Chinese } \\
\text { patent } \\
\text { medicine }\end{array}$ & 35 & 35 & $44.15 \pm 6.17$ & $44.39 \pm 6.41$ & $1 \mathrm{~m}$ & (1)(2) \\
\hline $\begin{array}{l}\text { Zhang Jianjun } \\
2017^{[32]}\end{array}$ & Random & $\begin{array}{l}\text { Xiangsha Liujunzi } \\
\text { Decoction }\end{array}$ & $\begin{array}{l}\text { Western } \\
\text { medicine }\end{array}$ & 47 & 47 & $52.3 \pm 3.4$ & $52.3 \pm 3.4$ & $8 w$ & (1)(2)(3) \\
\hline $\begin{array}{l}\text { Zhang Xiaoyu } \\
\qquad 2018^{[33]}\end{array}$ & Random & $\begin{array}{l}\text { Xiangsha Liujunzi } \\
\text { Decoction }\end{array}$ & $\begin{array}{l}\text { Western } \\
\text { medicine }\end{array}$ & 34 & 34 & $39.5 \pm 17.5$ & $39.5 \pm 15.5$ & $7 \mathrm{~d}$ & (1) \\
\hline $\begin{array}{c}\text { Zhao Yuren } \\
2019^{[34]}\end{array}$ & Random & $\begin{array}{l}\text { Xiangsha Liujunzi } \\
\text { Decoction }\end{array}$ & $\begin{array}{l}\text { Western } \\
\text { medicine }\end{array}$ & 67 & 67 & $50.81 \pm 5.46$ & $50.32 \pm 5.71$ & $12 \mathrm{w}$ & $\begin{array}{l}\text { (1) (4) (5) (6) } 7 \\
\text { (8) }\end{array}$ \\
\hline $\begin{array}{l}\text { Zhu Guojun } \\
2010^{[35]}\end{array}$ & Random & $\begin{array}{l}\text { Xiangsha Liujunzi } \\
\text { Decoction }\end{array}$ & $\begin{array}{l}\text { Western } \\
\text { medicine }\end{array}$ & 31 & 25 & $32-68$ & $31-69$ & $3 m$ & (1) \\
\hline $\begin{array}{l}\text { Peng Rentong } \\
2016^{[36]}\end{array}$ & Random & $\begin{array}{l}\text { Xiangsha Liujunzi } \\
\text { Decoction }\end{array}$ & $\begin{array}{l}\text { Western } \\
\text { medicine }\end{array}$ & 36 & 36 & $33-64$ & $35-65$ & $3 \mathrm{~m}$ & (1) \\
\hline $\begin{array}{l}\text { Luo Manhong } \\
\text { 2019 }\end{array}$ & Random & $\begin{array}{c}\text { Xiangsha Liujunzi } \\
\text { Decoction + Control } \\
\text { group }\end{array}$ & $\begin{array}{l}\text { Western } \\
\text { medicine }\end{array}$ & 50 & 50 & $46.1 \pm 8.7$ & $45.6 \pm 8.5$ & $8 w$ & $\begin{array}{l}\text { (1)(2)(3)(4) } 5 \\
\text { (9)(10) }\end{array}$ \\
\hline $\begin{array}{l}\text { Zhang Fang } \\
2019^{[38]}\end{array}$ & $\begin{array}{c}\text { Random } \\
\text { number table }\end{array}$ & $\begin{array}{c}\text { Xiangsha Liujunzi } \\
\text { Decoction + Control } \\
\text { group }\end{array}$ & $\begin{array}{l}\text { Western } \\
\text { medicine }\end{array}$ & 53 & 53 & $38.6 \pm 9.2$ & $37.9 \pm 9.9$ & $4 w$ & (1) \\
\hline $\begin{array}{l}\text { Liao Derong } \\
2015^{[39]}\end{array}$ & Random & $\begin{array}{c}\text { Xiangsha Liujunzi } \\
\text { Decoction + Control } \\
\text { group }\end{array}$ & $\begin{array}{l}\text { Western } \\
\text { medicine }\end{array}$ & 15 & 15 & $40.2 \pm 2.3$ & $40.2 \pm 2.3$ & $2 \mathrm{~m}$ & (1)(2) \\
\hline Chen Lili $2019^{[40]}$ & Random & $\begin{array}{c}\text { Xiangsha Liujunzi } \\
\text { Decoction + Control } \\
\text { group }\end{array}$ & $\begin{array}{l}\text { Western } \\
\text { medicine }\end{array}$ & 35 & 35 & $39.5 \pm 7.3$ & $44.6 \pm 6.4$ & $2 \mathrm{~m}$ & (1)(4) \\
\hline $\begin{array}{l}\text { Mao Yihui } \\
2020^{[41]}\end{array}$ & $\begin{array}{l}\text { Envelope } \\
\text { method }\end{array}$ & $\begin{array}{l}\text { Xiangsha Liujunzi } \\
\text { Decoction + Control } \\
\text { group }\end{array}$ & $\begin{array}{l}\text { Western } \\
\text { medicine }\end{array}$ & 50 & 50 & $55.7 \pm 4.6$ & $54.2 \pm 4.3$ & $2 \mathrm{~m}$ & (1) \\
\hline
\end{tabular}

T: Treatment group, C: Control group, -: Not reported, (1) Clinical total effective rate, (2) Recurrence rate, (3) Hp negative conversion, (4) Gastric pain symptom score, (5) Anorexia symptom score, (6) Loose stool symptom score, (7) Shortness of breath and laziness symptom score, (8) Piman discomfort symptom score, (9) SOD, (10) MDA.

\subsection{Basic Information Included in the Study}

The basic information of the included studies is shown in Table 1. Finally, 31 RCTs[11-41] were included, with a total of 2898 patients (1468 in the experimental group and 1430 in the control group). All studies reported that the baseline was comparable.

\subsection{Bias Risk Assessment Results}

The bias risk evaluation results are shown in Figure 3. All studies mentioned "random". 6 studies used "random number table" for randomization, 1 study used lottery grouping, and 1 study used envelope method, with low risk of bias. The 13 studies did not describe specific random methods. In the aspect of "implementation bias", one study used double-blind screening method with low risk of bias, and none of its studies described blinding. In terms of "follow-up bias", only one study reported the loss of follow-up (5 cases in the experimental group and 18 cases in the control group), which did not explain the method of dealing with the shedding data. The risk of bias was high and the outcome data were complete. All studies did not report results selectively, and no other bias was mentioned.

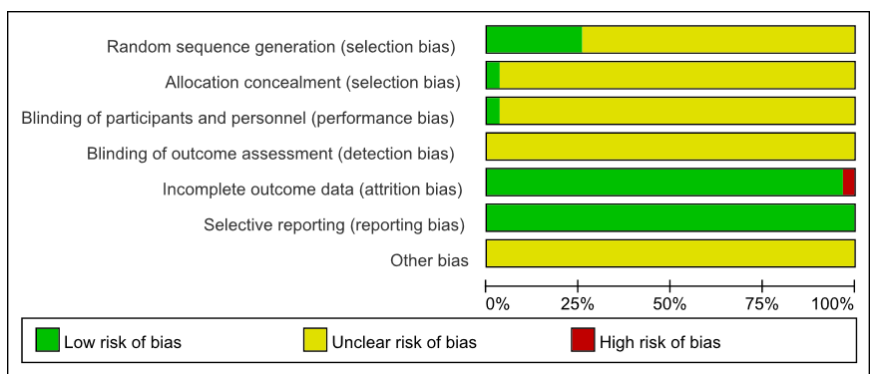

Figure 3: Proportion of projects included in studies at risk of bias

\subsection{Meta-analysis}

3.4.1 Clinical total effective rate 
31 studies[11-41] reported clinical total effective rate. Meta-analysis results of fixed effect model showed that the clinical total effective rate was statistically different among the studies [RR=1.31, 95\% CI $(1.26,1.36), \quad \mathrm{P}<0.00001]$, indicating that the clinical effective rate of Xiangsha Liujunzi Decoction in the treatment of CAG was better than that of Western medicine or Chinese patent medicine, as shown in Figure 4. The results of different intervention subgroups showed that the total effective rate of Xiangsha Liujunzi decoction group was higher than that of western medicine or Chinese patent medicine group and Xiangsha Liujunzi decoction group $[\mathrm{RR}=1.34,95 \% \mathrm{CI}(1.28,1.40), \mathrm{P}<0.00001]$. The total clinical effective rate of Xiangsha Liujunzi Decoction + control group VS western medicine or Chinese patent medicine group, Xiangsha Liujunzi Decoction + control group $[\mathrm{RR}=1.24,95 \% \mathrm{CI}(1.16,1.31), \mathrm{P}<0.00001]$ was higher than that of the control group, and the difference was statistically significant.

\begin{tabular}{|c|c|c|c|c|c|c|}
\hline \multirow[b]{2}{*}{ Study or Subgroup } & \multicolumn{2}{|c|}{ Experimental } & Contro & \multicolumn{3}{|r|}{ Risk Ratio } \\
\hline & \multirow{2}{*}{\multicolumn{6}{|c|}{$\begin{array}{l}\text { Events Total Events Total Weight } \mathrm{M} \text {-H, Fixed, } 95 \% \mathrm{Cl} \\
\text { coction group vs western medicine or Chinese patent medicine }\end{array}$}} \\
\hline 1.1.1 Xiangsha Liujunzi Decoction group vs western medicine or Chinese patent medicine & & & & & & \\
\hline Chen Li 2019 & 39 & 40 & 31 & 40 & $3.1 \%$ & $1.26[1.06,1.50]$ \\
\hline Du Jingfang 2012 & 42 & 48 & 30 & 48 & $3.0 \%$ & $1.40[1.10,1.79]$ \\
\hline Gao Meifu 2017 & 44 & 50 & 31 & 50 & $3.1 \%$ & $1.42[1.12,1.80]$ \\
\hline Lai Chan 2020 & 59 & 65 & 49 & 65 & $4.8 \%$ & $1.20[1.03,1.41]$ \\
\hline Lai Yu 2012 & 27 & 30 & 19 & 30 & $1.9 \%$ & $1.42[1.06,1.91]$ \\
\hline Li Haifeng 2014 & 29 & 31 & 19 & 31 & $1.9 \%$ & $1.53[1.14,2.05]$ \\
\hline Luo Yuanhan 2007 & 35 & 38 & 20 & 30 & $2.2 \%$ & $1.38[1.06,1.81]$ \\
\hline Ma Shihua 2011 & 42 & 48 & 30 & 48 & $3.0 \%$ & $1.40[1.10,1.79]$ \\
\hline Mao Pingan 2010 & 39 & 45 & 21 & 31 & $2.5 \%$ & $1.28[0.98,1.67]$ \\
\hline Peng Rentong 2016 & 32 & 36 & 27 & 36 & $2.7 \%$ & $1.19[0.95,1.48]$ \\
\hline Song Shixing 2017 & 38 & 40 & 26 & 40 & $2.6 \%$ & $1.46[1.15,1.85]$ \\
\hline Su Xiaoyun 2016 & 50 & 56 & 42 & 56 & $4.1 \%$ & $1.19[1.00,1.42]$ \\
\hline Tang Youming 2010 & 70 & 75 & 43 & 75 & $4.2 \%$ & $1.63[1.33,2.00]$ \\
\hline Wang Jianping 2013 & 52 & 70 & 31 & 70 & $3.1 \%$ & $1.68[1.25,2.26]$ \\
\hline Wang Xuemei 2014 & 35 & 38 & 25 & 38 & $2.5 \%$ & $1.40[1.09,1.79]$ \\
\hline Wang Yawei 2014 & 64 & 69 & 51 & 69 & $5.0 \%$ & $1.25[1.07,1.47]$ \\
\hline Wen Liqiong 2011 & 111 & 120 & 75 & 120 & $7.4 \%$ & $1.48[1.28,1.72]$ \\
\hline Zhang Changming 2018 & 34 & 35 & 29 & 35 & $2.9 \%$ & $1.17[1.00,1.38]$ \\
\hline Zhang Jianjun 2017 & 45 & 47 & 43 & 47 & $4.2 \%$ & $1.05[0.94,1.16]$ \\
\hline Zhang Xiaoyu 2018 & 32 & 34 & 26 & 34 & $2.6 \%$ & $1.23[1.00,1.51]$ \\
\hline Zhao Yuren 2019 & 61 & 67 & 50 & 67 & $4.9 \%$ & $1.22[1.04,1.43]$ \\
\hline Zhu Guojun 2010 & 29 & 31 & 17 & 25 & $1.9 \%$ & $1.38[1.04,1.83]$ \\
\hline Subtotal $(95 \% \mathrm{Cl})$ & & 1113 & & 1085 & $73.4 \%$ & $1.34[1.28,1.40]$ \\
\hline \\
\hline \multirow{2}{*}{\multicolumn{7}{|c|}{ 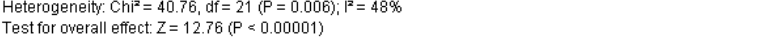 }} \\
\hline & & & & & & \\
\hline \multicolumn{7}{|c|}{ 1.1.2 Xiangsha Liujunzi Decoction + control group VS western medicine or Chinese patent medicine grol } \\
\hline Chen Lili 2019 & 33 & 35 & 27 & 35 & $2.7 \%$ & $1.22[1.00,1.49]$ \\
\hline Li Ning 2020 & 38 & 40 & 31 & 40 & $3.1 \%$ & $1.23[1.02,1.47]$ \\
\hline Liao Derong 2015 & 14 & 15 & 10 & 15 & $1.0 \%$ & $1.40[0.95,2.05]$ \\
\hline Liu Qiaoyi 2014 & 28 & 30 & 21 & 30 & $2.1 \%$ & $1.33[1.04,1.72]$ \\
\hline Luo Manhong 2019 & 46 & 50 & 38 & 50 & $3.8 \%$ & $1.21[1.02,1.44]$ \\
\hline Mao Yihui 2020 & 50 & 50 & 41 & 50 & $4.1 \%$ & $1.22[1.06,1.39]$ \\
\hline Wang Musheng 2008 & 43 & 45 & 25 & 35 & $2.8 \%$ & $1.34[1.07,1.66]$ \\
\hline Wang Shungin 2020 & 36 & 37 & 31 & 37 & $3.1 \%$ & $1.16[1.00,1.35]$ \\
\hline Zhang Fang 2019 & 50 & 53 & 42 & 53 & $4.1 \%$ & $1.19[1.02,1.39]$ \\
\hline Subtotal $(95 \% \mathrm{Cl})$ & & 355 & & 345 & $26.6 \%$ & $1.24[1.16,1.31]$ \\
\hline \multirow{2}{*}{\multicolumn{7}{|c|}{$\begin{array}{l}\text { Total events } \\
\text { Heterogeneity: } C h i^{2}=2.25, d f=8(P=0.97) ; P^{2}=0 \%\end{array}$}} \\
\hline & & & & & & \\
\hline \multicolumn{7}{|c|}{ 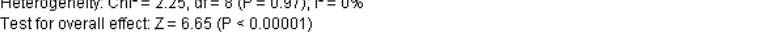 } \\
\hline Total $(95 \% \mathrm{Cl})$ & & 1468 & & 1430 & $100.0 \%$ & $1.31[1.26,1.36]$ \\
\hline & & & & & & \\
\hline Heterogeneity $C \mathrm{Ch}^{2}=44$. & $d f=30$ & $=0.06$ & $=32$ & & & \\
\hline & & & & & & \\
\hline
\end{tabular}

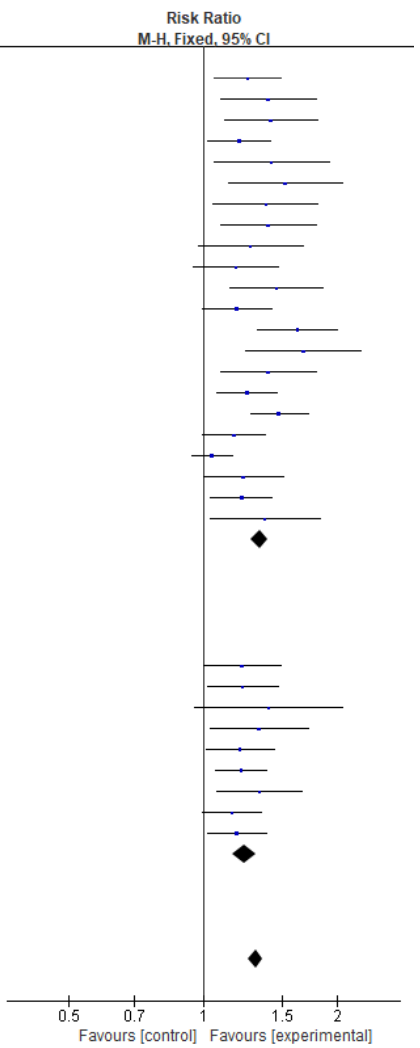

Figure 4: Meta-analysis of clinical total effective rate

\subsubsection{Recurrence rate}

8 studies[12,18,22,29,31,32,37,39] reported the recurrence rate. Meta-analysis of the fixed effect model showed that the recurrence rate was statistically different among the studies $[\mathrm{RR}=0.34,95 \% \mathrm{CI}(0.24,0.48), \mathrm{P}<0.00001]$, indicating that the recurrence rate of Xiangsha Liujunzi Decoction in the treatment of CAG was lower than that of Western medicine or Chinese patent medicine, as shown in Figure 5. The results of different intervention subgroups showed that the recurrence rate of Xiangsha Liujunzi decoction group was lower than that of western medicine or Chinese patent medicine group $[\mathrm{RR}=0.33,95 \% \mathrm{CI}(0.21,0.52), \mathrm{P}<0.00001]$. The recurrence rate $[\mathrm{RR}=0.37,95 \% \mathrm{CI}(0.22,0.61), \mathrm{P}<0.0001]$ of Xiangsha Liujunzi Decoction + control group VS western medicine or Chinese patent medicine group, Xiangsha Liujunzi Decoction + control group was lower than that of control group, the difference was statistically significant.

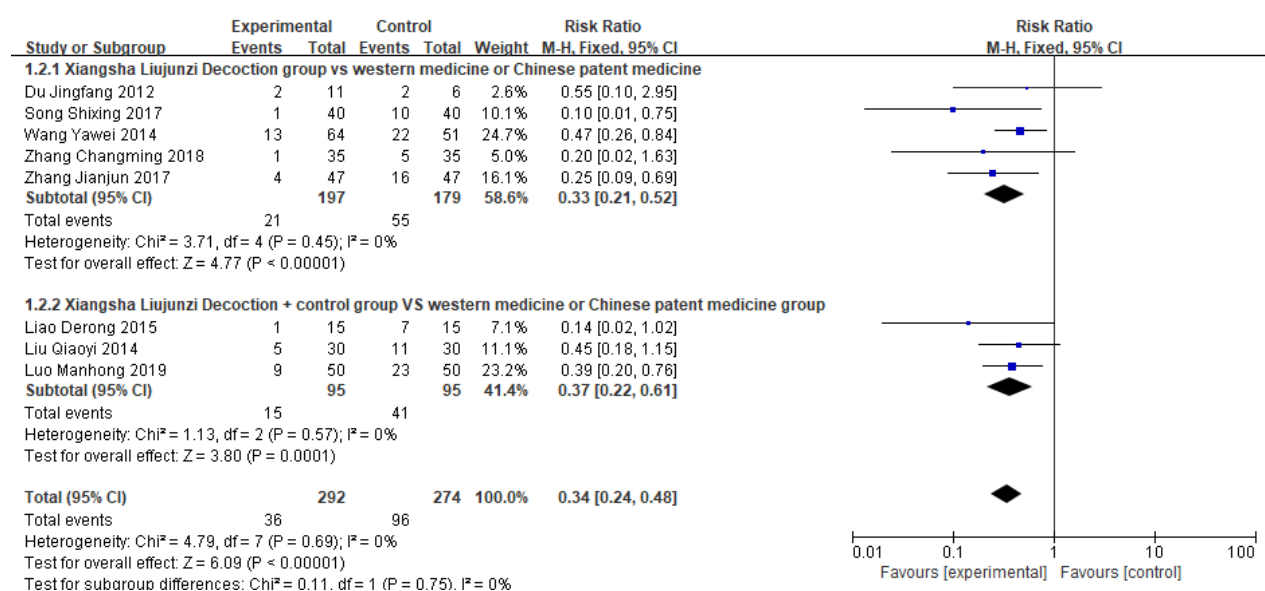

Figure 5: Meta-analysis of recurrence rate 


\subsubsection{Hp negative conversion}

6 studies[16,18,22,29,32,37] reported Hp negative conversion. Meta-analysis of fixed effect model showed that Hp negative conversion was statistically different among studies $[\mathrm{RR}=1.58,95 \% \mathrm{CI}(1.37,1.82), \mathrm{P}<0.00001]$, indicating that the Hp negative conversion of Xiangsha Liujunzi Decoction on CAG was better than that of Western medicine or Chinese patent medicine, as shown in Figure 6. The results of subgroups of different intervention measures showed that the Hp negative conversion rate in Xiangsha Liujunzi Decoction group vs western medicine or Chinese patent medicine group and Xiangsha Liujunzi Decoction group $[R R=1.80$, $95 \% \mathrm{CI}(1.45,2.23), \mathrm{P}<0.00001]$ was higher than that in the control group, and the difference was statistically significant. The Hp negative conversion of Xiangsha Liujunzi Decoction + control group VS western medicine or Chinese patent medicine group, Xiangsha Liujunzi Decoction + control group $[\mathrm{RR}=0.37,95 \% \mathrm{CI}(0.22,0.61), \mathrm{P}<0.0001]$ was higher than that of the control group, and the difference was statistically significant.

Study or Subgroup
$\begin{aligned} & \text { Experimental } \\ & \text { Events }\end{aligned}$ Total
Events

Figure 6: Meta-analysis of Hp negative conversion

\subsubsection{TCM symptom score}

\subsubsection{Gastric pain symptom score}

Xiangsha Liujunzi Decoction group vs western medicine or Chinese patent medicine subgroup were included in 6 studies[11,14,15,23,24,34]. The results of meta-analysis of random effect model showed that there was significant difference in gastric pain symptom score between the 2 groups $\quad[\mathrm{MD}=-0.77, \quad 95 \% \mathrm{CI}(-1.03,-0.51), \quad \mathrm{P}<0.00001]$, indicating that Xiangsha Liujunzi Decoction was superior to western medicine or Chinese patent medicine in improving gastric pain symptoms in CAG. A total of 3 studies[27,37,40] were included in the Xiangsha Liujunzi Decoction + the control group drug vs western medicine or Chinese patent medicine subgroup. The results of random effect model Meta-analysis showed that there was a statistically significant difference in the scores of gastric pain symptoms between the 2 groups $[\mathrm{MD}=-0.62,95 \% \mathrm{CI}(-0.86,-0.37), \quad \mathrm{P}<0.00001]$, indicating that the improvement effect of Xiangsha Liujunzi Decoction combined with the control group on gastric pain symptoms of CAG in traditional Chinese medicine was better than that of western medicine or Chinese patent medicine, as shown in Figure 7.

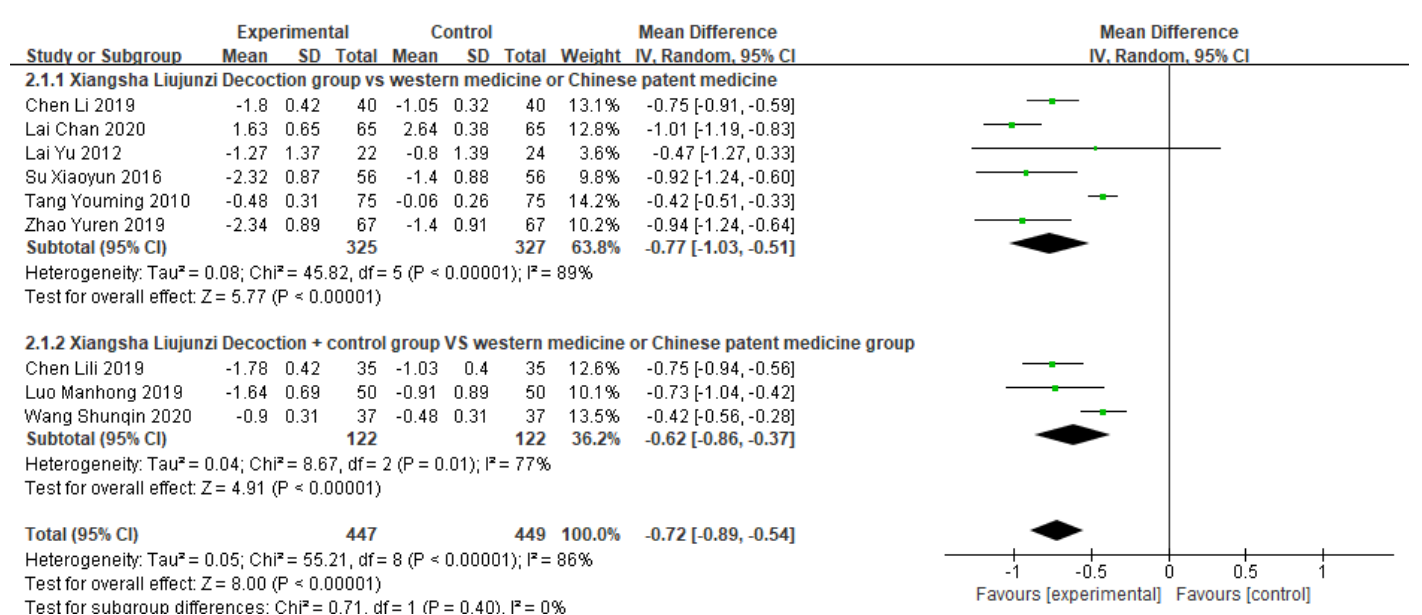

Figure 7: Meta-analysis of gastric pain symptom score

\subsubsection{Anorexia symptom score}

Xiangsha Liujunzi Decoction VS Western medicine or Chinese patent medicine sub-group were included in 5 studies[14,15,23,24,34], random effect model Meta-analysis showed that anorexia symptom score between the two groups was statistically significant $[\mathrm{MD}=-0.59,95 \% \mathrm{CI}(-0.77,-0.41)$,
$\mathrm{P}<0.00001]$, indicating that Xiangsha Liujunzi Decoction on CAG anorexia symptom improvement is better than western medicine or Chinese patent medicine, Figure 8. The addition and subtraction of Xiangsha Liujunzi Decoction + the control group drug VS western medicine or Chinese patent medicine subgroup only included 1 study[37], which could not be analyzed by Meta-analysis. 


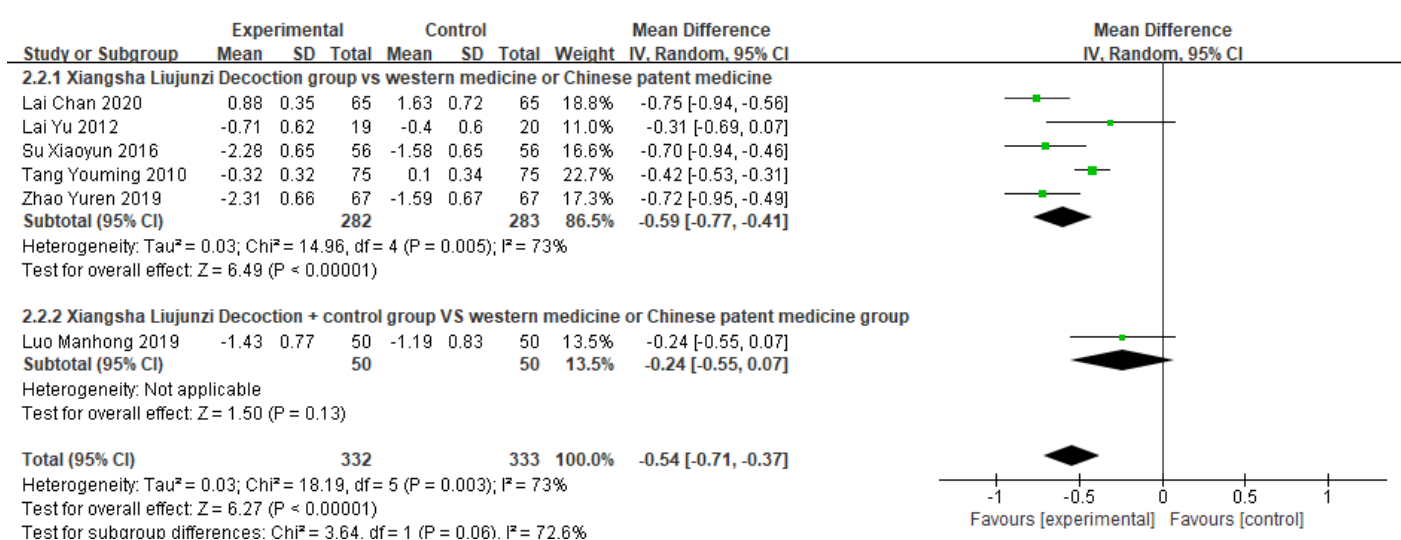

Figure 8: Meta-analysis of anorexia symptom score

\subsubsection{Loose stool symptom score}

Xiangsha Liujunzi Decoction vs western medicine or Chinese patent medicine subgroup were included in 5 studies[14,15,23,24,34]. The results of meta-analysis of random effect model showed that there was significant difference in symptom scores of loose stool between the 2 groups $[\mathrm{MD}=-0.52,95 \% \mathrm{CI}(-0.83,-0.20), \mathrm{P}=0.001]$, indicating that Xiangsha Liujunzi Decoction was superior to Western medicine or Chinese patent medicine in improving symptoms of loose stool in CAG, as shown in figure 9. Xiangsha Liujunzi Decoction + control group vs western medicine or Chinese patent medicine subgroup was not included in the study.

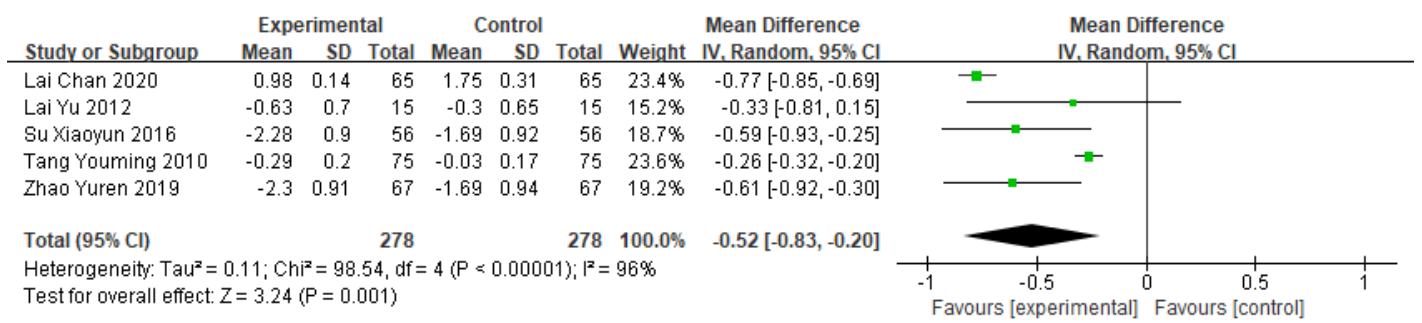

Figure 9: Meta-analysis of loose stool symptom score

\subsubsection{Shortness of breath and laziness symptom score}

Xiangsha Liujunzi Decoction vs western medicine or Chinese patent medicine subgroup were included in 5 studies[14,15,23,24,34]. The results of meta-analysis of random effect model showed that the shortness of breath and laziness symptom score was statistically significant between the 2 groups [MD=-0.45, 95\% CI(-0.73,-0.16), $\mathrm{P}=0.002]$, indicating that Xiangsha Liujunzi Decoction was superior to western medicine or Chinese patent medicine in improving the symptoms of shortness of breath in $\mathrm{CAG}$, as shown in figure 10. Xiangsha Liujunzi Decoction + control group vs western medicine or Chinese patent medicine subgroup was not included in the study.

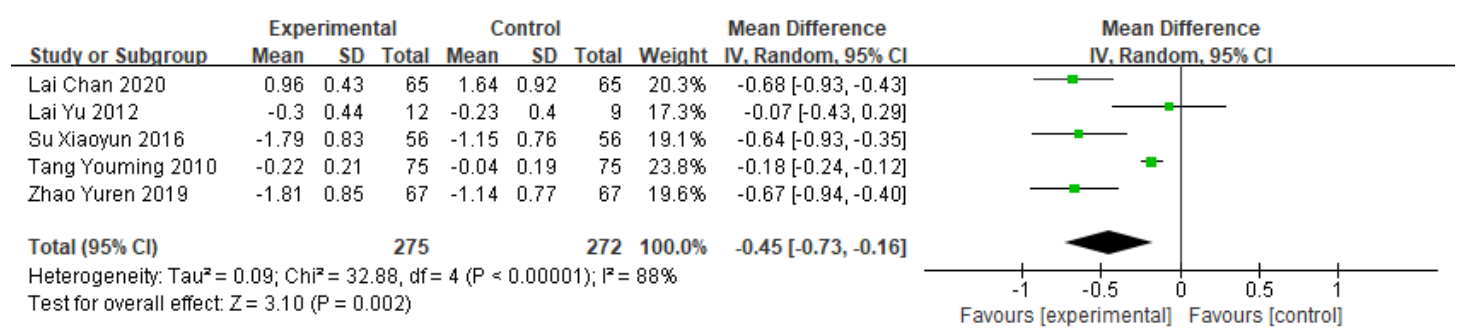

Figure 10: Meta-analysis of shortness of breath lazy speech symptom score

\subsubsection{Piman discomfort symptom score}

Xiangsha Liujunzi Decoction vs western medicine or Chinese patent medicine subgroup were included in 4 studies[15,23,24,34]. The results of meta-analysis of random effect model showed that the symptom score of fullness and discomfort was statistically significant between the 2 groups
$[\mathrm{MD}=-0.75,95 \% \mathrm{CI}(-1.10,-0.41), \mathrm{P}<0.0001]$, indicating that Xiangsha Liujunzi Decoction was superior to western medicine or Chinese patent medicine in improving the symptoms of fullness and discomfort in CAG, as show Figure 11. Xiangsha Liujunzi Decoction + control group vs western medicine or Chinese patent medicine subgroup was not included in the study. 


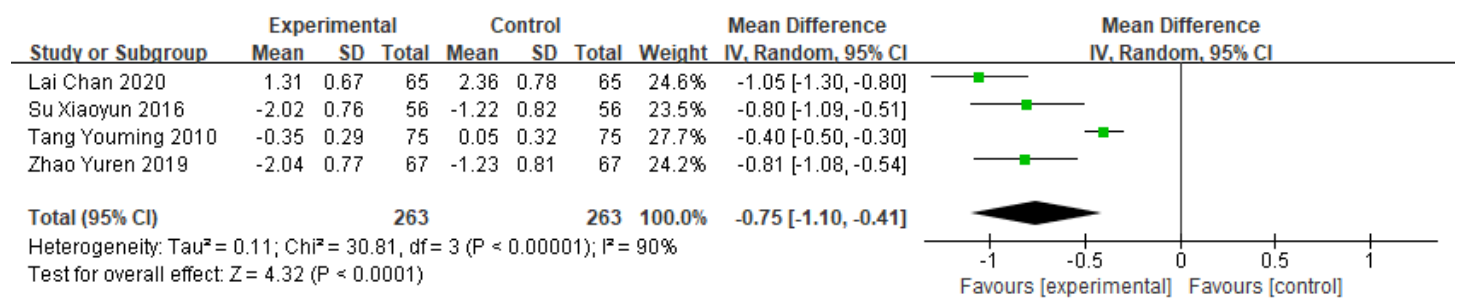

Figure 11: Meta-analysis of piman discomfort symptom score

\subsubsection{Oxidative stress response index level}

\subsubsection{SOD}

Xiangsha Liujunzi Decoction + control group drug vs western medicine or Chinese patent medicine sub-group were included in 2 studies[17,37]. The results of random effect model Meta-analysis showed that there was a statistically significant difference in SOD between the 2 groups $[\mathrm{MD}=22.49,95 \% \mathrm{CI}(12.65,32.32), \mathrm{P}<0.00001]$, indicating that compared with western medicine or Chinese patent medicine, Xiangsha Liujunzi Decoction combined with control group drug increased SOD level more significantly, as shown in Figure. 12. Xiangsha Liujunzi Decoction vs western medicine or Chinese patent medicine subgroup was not included in the study.

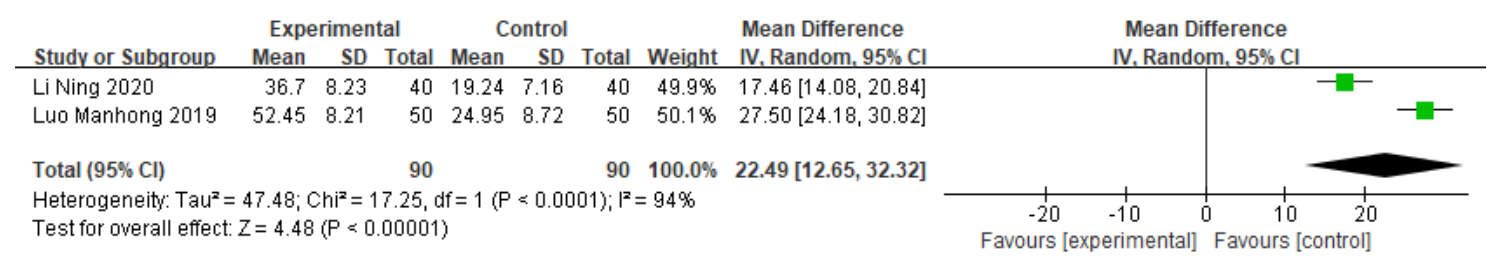

Figure 12: Meta-analysis of SOD

\subsubsection{MDA}

Xiangsha Liujunzi Decoction + control group vs western medicine or Chinese patent medicine sub-group were included in 2 studies[17,37]. The results of random effect model meta-analysis showed that there was no significant difference in MDA between the 2 groups

\begin{tabular}{|c|c|c|c|c|c|c|c|c|c|c|c|}
\hline \multirow[b]{2}{*}{ Study or Subgroup } & \multicolumn{3}{|c|}{ Experimental } & \multicolumn{3}{|c|}{ Control } & \multirow{2}{*}{\multicolumn{2}{|c|}{$\begin{array}{c}\text { Mean Difference } \\
\text { Weight IV, Random, } 95 \% \mathrm{Cl} \\
\end{array}$}} & \multirow{2}{*}{\multicolumn{3}{|c|}{$\begin{array}{c}\text { Mean Difference } \\
\text { IV, Random, } 95 \% \mathrm{Cl}\end{array}$}} \\
\hline & Mean & SD & Total & Mean & SD & Total & & & & & \\
\hline Li Ning 2020 & -14.69 & 3.61 & 40 & -8.69 & 3.52 & 40 & $48.5 \%$ & $-6.00[-7.56,-4.44]$ & - & & \\
\hline Luo Manhong 2019 & -2.12 & 0.77 & 50 & -0.59 & 0.59 & 50 & $51.5 \%$ & $-1.53[-1.80,-1.26]$ & & & \\
\hline Total $(95 \% \mathrm{Cl})$ & & & 90 & & & 90 & $100.0 \%$ & $-3.70[-8.07,0.68]$ & $\mathbf{\square}$ & & \\
\hline \multicolumn{12}{|c|}{ 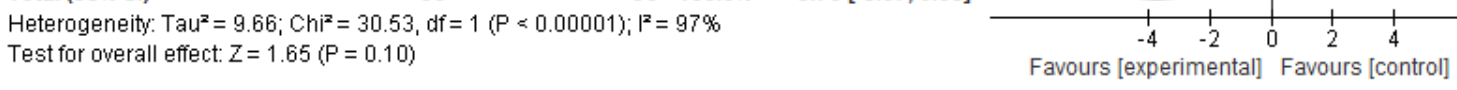 } \\
\hline
\end{tabular}

Figure 13: Meta-analysis of MDA

\subsection{Sensitivity Analysis}

Sensitivity analysis was conducted by eliminating individual studies one by one, and no significant change in the merged results after elimination suggested that the Meta-analysis results were stable.

\section{Discussion}

CAG is a common clinical disease. Studies have shown that the main causes of CAG are Hp infection, bile reflux, autoimmune factors, genetic factors and poor $\operatorname{diet}[42,43]$, and the first factor is HP infection, which is closely related to the risk of intestinal metaplasia and cancer. Traditional Chinese medicine believes that CAG is mainly related to many factors, such as weakness of spleen and stomach, irregular diet, emotional disorders, drugs, external evils and so on. These factors can damage the spleen and stomach, cause transportation disorders, and abnormal lifting, which are manifested as stomach pain, fullness and other symptoms. It belongs to the category of "epigastric pain", "fullness",
$[\mathrm{MD}=-3.70,95 \% \mathrm{CI}(-8.07,0.68, \quad \mathrm{P}=0.10)]$, indicating that Xiangsha Liujunzi Decoction combined with the control group significantly decreased MDA level compared with western medicine or Chinese patent medicine, as shown in Figure. 13. Xiangsha Liujunzi Decoction vs western medicine or Chinese patent medicine subgroup was not included in the study.

Favours [experimental] Favours [control] "anti-acid", "noisy" in traditional Chinese medicine, and its pathological nature is always deficient in origin and excessive in superficiality. Xiangsha Liujunzi Decoction is composed of Ginseng, Atractylodes, Poria, Pinellia, Pericarpium Citri Reticulatae, Amomum villosum, Amomum villosum and Glycyrrhiza uralensis. Its efficacy is to benefit qi and harmonize stomach and promote qi stagnation. Its mechanism of action on CAG is mainly to protect gastric mucosa, reduce inflammation, improve gastric mucosal secretion, regulate gastrointestinal motility, eradicate HP and block the development of cancer, and its side effects are less, curative effect is exact and safe. Although three related systematic reviews have been published in the past, the number of studies included is small, the methodological quality is low, and the outcome indicators are relatively single.

In this study, 31 clinical randomized controlled trials were included. The results of Meta-system analysis showed that Xiangsha Liujunzi Decoction could better improve the pathological status of patients, enhance clinical efficacy, reduce recurrence rate and oxidative stress response, and increase Hp negative conversion rate compared with Western 
medicine or Chinese patent medicine in the treatment of CAG. The effect was more prominent in improving clinical symptoms such as stomach pain, anorexia and eating less, loose stool, shortness of breath and laziness, and stuffiness.

Limitations of this study: 1) Interventions included in the study, such as drug combination types, course of treatment and efficacy evaluation criteria, are quite different. Limited by the number of studies, further subgroup analysis cannot be carried out to affect the accuracy of the results ; 2) Most of the included studies did not carry out allocation concealment and blind method, which may lead to bias in selection and implementation ; 3) The number of samples included in the study was small, and most of them did not have clear follow-up time or follow-up time was short. The evaluation of late indicators such as $\mathrm{Hp}$ eradication and recurrence rate was lacking, and the long-term effect was not clear.

In summary, the existing evidence shows that Xiangsha Liujunzi Decoction alone or combined with Western medicine or Chinese patent medicine in the treatment of CAG can significantly improve the total clinical effective rate and $\mathrm{Hp}$ negative rate, reduce the recurrence rate and oxidative stress response, and improve the clinical symptoms such as stomachache, anorexia, loose stools, shortness of breath, laziness and discomfort. Limited by the quantity and quality of included studies, the above conclusions need to be verified by more high-quality studies.

\section{References}

[1] Botezatu A, Bodrug N. Chronic atrophic gastritis: an update on diagnosis[J]. Med Pharm Rep, 2021, 94(1): 7-14.

[2] Shah S C, Piazuelo M B, Kuipers E J, et al. AGA Clinical Practice Update on the Diagnosis and Management of Atrophic Gastritis: Expert Review[J]. Gastroenterology, 2021, 161(4): 1325-1332.e7.

[3] Rodriguez-Castro K I, Franceschi M, Noto A, et al. Clinical manifestations of chronic atrophic gastritis[J]. Acta Biomed, 2018, 89(8-s): 88-92.

[4] Lahner E, Carabotti M, Annibale B. Treatment of Helicobacter pylori infection in atrophic gastritis[J]. World J Gastroenterol, 2018, 24(22): 2373-2380.

[5] Du Y, Bai Y, Xie P, et al. Chronic gastritis in China: a national multi-center survey[J]. BMC Gastroenterol, 2014, 14: 21.

[6] Sharma P K, Suri T M, Venigalla P M, et al. Atrophic gastritis with high prevalence of Helicobacter pylori is a predominant feature in patients with dyspepsia in a high altitude area[J]. Trop Gastroenterol, 2014, 35(4): 246-51.

[7] Zhou Jianzhong, Chen Zemin, Gu Beihai.Chronic gastritis diagnosis, syndrome differentiation and curative effect standard of integrated traditional Chinese and western medicine (trial scheme) [J]. Chinese Journal of Integrated Traditional and Western Medicine, 1990(05): 318-319.

[8] The Minister of Health of the People's Republic of China. Guiding Principles for Clinical Research on New Drugs of Traditional Chinese Medicine (Trial) [M]. Beijing: China Pharmaceutical Science and Technology Press, 2002: 124-129.
[9] Tang Xudong, Li Zhenhua, Li Baoshuang, et al.Chronic gastritis diagnosis and treatment guidelines $[\mathrm{J}]$. Chinese Medicine Modern Distance Education of China, 2011, 9(10): 123-125.

[10] Zhang Shengsheng, Li Gangou, Tang Xudong, et al.Consensus of TCM diagnosis and treatment of chronic atrophic gastritis [J]. Journal of Traditional Chinese Medicine, 2010, 51(08): 749-753.

[11] Clinical Observation on 40 Cases of Chronic Atrophic Gastritis Treated with Modified Xiangsha Liujunzi Decoction [J]. Famous Doctors, 2019(06): 242.

[12] Du Jingfang. Xiangsha Liujunzi Decoction in the Treatment of Chronic Atrophic Gastritis Clinical Research [J]. Acta Chinese Medicine, 2012, 27(09): 1181-1182.

[13] Gao Meifu. Clinical Analysis of Xiangsha Liujunzi Decoction in Treating Chronic Atrophic Gastritis [J]. China Health Care Nutrition, 2017, 27(5): 147.

[14] Lai Yu, Wu Hongjin, Xu Baocai. Clinical Observation on 30 Cases of Chronic Atrophic Gastritis Treated with Modified Xiangsha Liujunzi Decoction [J]. Journal of Hebei Traditional Chinese Medicine and Pharmacology, 2012, 27(04): 18-19.

[15] Lai Chan, Hong Zhujun. Mechanism and efficacy of Xiangsha Liujunzi Decoction in the treatment of chronic atrophic gastritis of spleen-stomach deficiency-cold type [J]. Cardiovascular Disease Journal Of integrated traditional Chinese and Western Medicine, 2020, 8(23): 139-162.

[16] Li Haifeng, Wang Limei. Clinical Observation on 62 Cases of Chronic Atrophic Gastritis Treated with Modified Xiangsha Liujunzi Decoction [J]. Pharmacology and Clinics of Chinese Materia Medica, 2014, 30(06): 179-180.

[17] Li Ning. Effect of Xiangsha Liujunzi Decoction on Oxidative Stress, PG I and PG II in Patients with Chronic Atrophic Gastritis [J]. Modern Medicine and Health Research Electronic Journa, 2020, 4(13): 74-76.

[18] Liu Yiqiao. Effect of Xiangsha Liujunzi Decoction on 30 Cases of Chronic Atrophic Gastritis [J]. Nei Mongol Journal of Traditional Chinese Medicine, 2014, 33(26): 12-13.

[19] Luo Yuanhan, Li Ping. 38 Cases of Chronic Atrophic Gastritis Treated with Xiangsha Liujunzi Decoction [J]. Jilin Journal of Traditional Chinese Medicine, 2007, 27(8): 28-29.

[20] Ma Shihua, Wu Jie. Therapeutic effect of Xiangsha Liujunzi Decoction on 48 cases of chronic atrophic gastritis [J]. Chinese Journal of Modern Drug Application, 2011, 5(16): 86-87.

[21] Mao Pingan, Zheng Songming, Wang Changxiong, et al. 45 Cases of Chronic Atrophic Gastritis with Deficiency of Spleen and Stomach Treated with Xiangsha Liujunzi Decoction [J]. Shandong Journal of Traditional Chinese Medicine, 2010(8).

[22] Song Shixing, Song Wanxun. Effect analysis of Xiangsha Liujunzi Decoction on chronic atrophic gastritis $[\mathrm{J}]$. Electronic Journal of Clinical Medical Literature, 2017, 4(82): 16115-16116.

[23] Su Xiaoyun. Clinical Observation on 56 Cases of Chronic Atrophic Gastritis of Spleen and Stomach Deficiency Type Treated with Xiangsha Liujunzi 
Decoction [J]. Chinese Journal of Ethnomedicine and Ethnopharmacy, 2016, 25(18): 84-85+87.

[24] Tang Youming. Clinical Observation on Xiangsha Liujunzi Pill in Treating 75 Cases of Chronic Atrophic Gastritis of Spleen and Stomach Deficiency Cold Type [J]. Yunnan Journal of Traditional Chinese Medicine and Materia Medica, 2010, 31(12).

[25] Wang Jianping, Wei Pinkang. Clinical Observation on 70 Cases of Chronic Atrophic Gastritis Treated with Xiangsha Liujunzi Decoction [J]. Guiding Journal of Traditional Chinese Medicine and Pharmacy, 2013, 19(05): 43-44.

[26] Wang Musheng, Xiong Fengbao. Modified Xiangsha Liujunzi Decoction in the treatment of chronic atrophic gastritis curative effect observation [J]. Practical clinical medicine, 2008(03): 40-41.

[27] Wang Shunqin, Wang Jingwen. Effect of Modified Xiangsha Liujunzi Decoction on Chronic Atrophic Gastritis with Helicobacter Pylori Infection [J]. Gansu Science and Technology, 2020, 36(21): 117-119.

[28] Wang Xuemei. Clinical Analysis of 76 Cases of Chronic Atrophic Gastritis Treated with Modified Xiangsha Liujunzi Decoction [J]. Clinical Journal of Chinese Medicine, 2014, 6(32): 116-117.

[29] Wang Yawei. 69 Cases of Chronic Atrophic Gastritis Treated with Xiangsha Liujunzi Decoction [J]. China Pharmaceuticals, 2014, 23(02): 82-83.

[30] Wen Liqiong. Clinical Observation on 120 Cases of Chronic Atrophic Gastritis Treated by Traditional Chinese Medicine [J]. Clinical Journal of Chinese Medicine, 2011, 3(18): 97.

[31] Zhang Changming. Effect analysis of Xiangsha Liujunzi Decoction on chronic atrophic gastritis [J]. Electronic Journal of Clinical Medical Literature, 2018, 5(34): $158+160$.

[32] Zhang Jianjun. Clinical Study on Modified Xiangsha Liujunzi Decoction for Chronic Atrophic Gastritis of Spleen and Stomach Deficiency Cold Syndrome [J]. Chinese Community Doctors, 2017, 33(02): 106-107.

[33] Zhang Xiaoyu, Wang Hai. Clinical Effect of Xiangsha Liujunzi Decoction on Chronic Atrophic Gastritis of Spleen and Stomach Deficiency Type [J]. World Latest Medicine Information, 2018, 18(83): 157+163.

[34] Zhao Yuren. Modified Xiangsha Liujunzi Decoction in Treating Chronic Atrophic Gastritis of Spleen and Stomach Deficiency Type [J]. Shenzhen Journal of Integrated Traditional Chinese and Western Medicine, 2019, 29(23): 65-66.

[35] Zhu Guojun. 31 Cases of Chronic Atrophic Gastritis Treated with Xiangsha Liujunzi Decoction [J]. Chinese Community Doctors (Medicine), 2010, 12(30): 119.

[36] Peng Rentong. Clinical Study on Xiangsha Liujunzi Decoction in Treating Chronic Atrophic Gastritis [J]. Henan Traditional Chinese Medicine, 2016, 36(02): 316-318.

[37] Luo Manhong. Clinical Observation on Treating Chronic Atrophic Gastritis with Modified Xiangsha Liujunzi Decoction Combined with Triple Therapy [J]. Guangzhou medical journal, 2019, 50(01): 28-32.

[38] Zhang Fang, Kang Chuanzhe, Hu Xiaoyi, et al. Effect of Xiangsha Liujun Pill Combined with Pantoprazole on Chronic Atrophic Gastritis [J]. Evaluation and Analysis of Drug-Use in Hospitals of China, 2019, 19(7).
[39] Liao Derong. Effect of Xiangsha Liujunzi Decoction Combined with Omeprazole on Chronic Atrophic Gastritis [J]. Medical Information, 2015, 28(50): 215-216.

[40] Chen Lili. Clinical Observation of Xiangsha Liujunzi Decoction Combined with Western Medicine in the Treatment of Chronic Atrophic Gastritis [J]. Guangming Journal of Chinese Medicine, 2019, 34(18): 2834-2836.

[41] Mao Yihui. Application Value of Xiangsha Liujunzi Decoction Combined with Western Medicine in the Treatment of Chronic Atrophic Gastritis [J]. Diet science: second half of the month, 2020(6).

[42] Ye M, Cheng J, Jin D, et al. Systematic review and meta-analysis based on the composition of risk factors of chronic atrophic gastritis under gastroscopy detection[J]. Ann Palliat Med, 2021, 10(9): 9742-9751.

[43] Li Y, Xia R, Zhang B, et al. Chronic Atrophic Gastritis: A Review[J]. J Environ Pathol Toxicol Oncol, 2018, 37(3): 241-259. 